\title{
The Essence of the Universe and Humankind
}

\author{
Samo Liu',2 \\ ${ }^{1}$ HMR Technology Holdings Group, Jinan, China \\ ${ }^{2}$ University of Science and Technology Beijing, Beijing, China \\ Email: samo945@126.com
}

How to cite this paper: Liu, S. M. (2020). The Essence of the Universe and Humankind. Open Journal of Philosophy, 10, 316330.

https://doi.org/10.4236/ojpp.2020.103021

Received: June 30, 2020

Accepted: August 3, 2020

Published: August 6, 2020

Copyright $\odot 2020$ by author(s) and Scientific Research Publishing Inc. This work is licensed under the Creative Commons Attribution International License (CC BY 4.0).

http://creativecommons.org/licenses/by/4.0/

(c) (i) Open Access

\begin{abstract}
The Essence of the three-dimensional Universe came from the energy from the multi-dimensional void, deprived of all matters. Dimensions above the three-dimensional Universe postulated in the M-theory are all abstract mathematical dimensions, and they could be grouped into the zero-dimensional Universe. The present known Universe is probably the highest dimension and it is one of the infinite numbers of pocket Universe floating in the zero-dimensional kong, or void. Matters, including humans, are the products from the combination of energy and materials. What sets humans apart from the rest of the living matters is the difference in the intelligent energy bestowed during the initial stage in the creation of human cells.
\end{abstract}

\section{Keywords}

Multi-Dimensional Universe, Zero-Dimensional Universe, Rational Perceptual Cognition, Dialectics, Oriental Knowledge, Intelligent Energy, Fundamental Energy, Energy, Matter

\section{Introduction to the Basis and Foundations of Reflection}

The theory of relativity, both the special and general relativity postulated by Albert Einstein (Germany, 1879-1955), is a very important theory that finally pieced together the relationship between materials in the three-dimensional Universe and space-time (Einstein, 2018). Humankind then took steps further with more discoveries in theoretical physics such as the M-theory proposed by Edward Witten, or the fundamentals of quantum mechanics that study the essence of materials, including that of humans. Arguably, the advent of modern physics is the finest achievement of humankind in recognition of the three-dimensional Universe.

However, when reflecting on the Essence of the entire Universe, the author raised some questions on the current model of space and time in modern phys- 
ics, where the theory of relativity failed to explain quantum behaviors. Subsequently, by incorporating oriental knowledge (later called Eastern philosophy) and the core philosophical ideologies of both the Western and Eastern philosophies from the Axial Age, a new way of thinking based on perceptual cognition came to light. Following the introducing works previously published (Liu, 2017; Liu, 2019; Liu, 2020), this paper would like to further discuss the Essence of the Universe by tentatively putting together a unified theory that can bridge the modern physics to philosophical ideologies from the ancient time, with more details in a work in progress, a book with around 500,000 words with Scientific Research. This paper will first introduce the Eastern concepts in perceptions and space, followed by a brief overview of space-time in the perceptive of modern physics, and an exploration of some important theories in modern theoretical physics. Finally, the paper will discuss the essence and origin of humankind and the Universe by incorporating notions from modern physics and classical Eastern philosophies. The ultimate goal of this paper is to give rise to a reflection on the peaceful future of humanity.

\section{Rational Perceptual Cognition and Immersive Concentration}

In ancient times, some philosophers once regarded the perceptual cognition of humankind as an irrational thing (Thilly, 1914). The Universe bestowed the rational perceptual cognition, a powerful information gathering system to the humankind when it emerged from particles. Another name to this rational perceptual cognition is immersive concentration.

All scientific inventions and discoveries, especially the fate-changing ones, require a tremendous level of immersive concentration in the reflection of the subject to develop concepts and theories, and then the appropriate action to realize the idea. There is no room for mistakes or carelessness during the entire process and its verification, which one at a time, would necessitate the full concentration of a great number of scientists. This example is the explicit manifestation of an immersive concentration.

The passive immersive concentration focuses on the human's mind, but to achieve this degree of concentration is extremely difficult (Porter, 2018). The passive immersive concentration often acts on parts of the human body, or sometimes on the entire body, rather than acts on any specific action. Another crucial condition to achieve a functioning passive immersive concentration is a total state of serenity that is free from the influence of materialistic desires, on top of peace of the surrounding environment. Whenever one enters this state of mind, one will perceive the Universe surrounding him and develop this passive rational perceptual cognition, the most powerful information acquiring system bestowed to humans by the Universe. However, its presence diminishes with the increasing outside influences such as materialistic desires, but it remains intrinsic to the human body.

During the Axial Age in human history, numerous great philosophers, ideolo- 
gists, and saints appeared both in the Eastern and Western cultural spheres. Amongst the most famous of them are Laozi (c. 571 - c. 471 BC), Gautama Buddha (c. 565 - c. 486 BC), Confucius (c. 551 - c. 479 BC), Mozi (c. 476 - c. 390 BC), Thales of Miletus (c. 624 - c. 546 BC), Parmenides of Elea (c. 515 - c. 550 BC), Pythagoras of Samos (c. 580 - c. 490 BC), Socrates (c. 470 - c. 399 BC) and Aristotle (c. 384 - c. 322 BC) (Hu, 2012; Thilly, 1914).

When describing this significant period, numerous historians tend to analyze the ideologies of these saints using perspectives and knowledge acquired from modern science using pure reason and logic. Because of this biased perspective, modern researchers often view ideologies from this period as metaphysics or pseudo-science. The reality, however, might be very different.

The great saints in the Axial Age used this rational perceptual cognition to realize the Essence of the Universe and humankind. They subsequently developed that enlightenment into ideologies that passed down throughout the generations. Since the Axial Age, this rational perceptual cognition has been practicing around the world, particularly in Eastern cultures, which was also a cause of the continuity of Eastern knowledge throughout history. Rational perceptual cognition of the Universe is one of the methods of self-cultivation, and one can achieve this by holding perseverance and firm belief. However, it is important to understand that this cognition can have errors, depending on the serenity of the surrounding environment, where the degree of interference is inversely proportional to the degree of serenity. Today, with the progress of scientific methods, to test the exactitude of the perceptual cognition, practitioners could record their passive cognition and verify it using analytical statistics.

\section{Space-Time}

\subsection{Space}

Every material in the three-dimensional Universe is made from particles encompassed with real matter, with a geometrical shape and volume in all three dimensions. Therefore, humans naturally believe that our space is three-dimensional. In the Universe, the material defines the dimension of its harboring space, and it came from human perception of its surrounding space. For example, materials such as a car, or humans are beings of three dimensions. Thus, they occupy three-dimensional space. On the other hand, a shadow is a two-dimensional being, and it harbors in a two-dimensional space. Finally, the border of a shadow is a one-dimensional entity, so it occupies the one-dimensional space.

However, human perception could be deceptive, because the Universe human lives in are not three-dimensional, or any other single dimension, but rather composed by a combination of multiple geometrical dimensions, from zero, one, two, to three-dimension, to which the author names it the dimension of existence. In this dimension of existence, the notions of one, two, or three-dimension are a reflection of the state of existence of any material it describes. 
Contrarily to mass, energy is an existence from the void in a way that it does not occupy any geometrical dimension in the space. Therefore, it can be arbitrarily considered an existence of zero dimension. By arguing using the dialectics of yin and yang, the author names this space, where energy exists as the zero positive space, denoted $0^{+}$. In this space, matters do not exist, just as described in the works of the German polymath Gottfried Leibniz (Leibniz, 2001). According to Leibniz, in the null space, any points are indifferent (Leibniz, 2001). The description of the null space is similar to what the Chinese philosophy has described: big without a border, small without a limit (Hu, 2012). To conform with the M-theory, this zero positive dimension is equivalent to the fourth dimension, and the author names it the null dimension. The null dimension can be regarded as a four-dimensional space-time coordinate system described in Einstein's theory with time set as zero. In this zero positive dimension, "genes" constitute the three-dimensional materials, similarly to Fermions that constitute all atoms. This "gene" would have no notions of time, matter, nor dimensions, and it can be called the fundamental energy.

From the dialectics of yin and yang, the void where information exists is also the zero space, contrarily to the space where energy exists, this space is the zero negative space, denoted the $0^{-}$, to distinguish from the zero positive dimension where energy exists. Time in this dimension is also zero. Following the sequence in the M-theory, this space proposed by the author is equivalent to the geometrical five-dimension and the author names it the dimension of change. In this dimension of change, the energy it encompasses is called the intelligent energy. This intelligent energy, in turn, is the cause for the creation of three-dimensional materials, similar to the Higgs boson in quantum mechanics.

The concept of the fundamental and intelligent energy, which respectively represents energy and information, came from the Eastern philosophical ideologies of "the myriad things came from the void (kong)" and "the myriad things came from the $q i$ of yin and yang" (Hu, 2012). The concept of $q i$ is described here as an energy concept. In turn, this $q i$ of yin and yang came from wuji, another concept of energy that can classify as the six-dimensional space from the sequencing of M-theory. This six-dimension can also refer to as the dimension of wuji or dao. If viewed from a scientific perspective, this concept is similar to that of quantum entanglement, such that the fundamental and intelligent energy or the zero positive and zero negative dimension cannot be described independently from each other.

The above concept was developed by combining the achievements of modern physics with Eastern philosophy, also known as oriental knowledge and philosophical ideologies from Ancient Greece. In addition, philosophical methods of dialectics materialism and logical reasoning were also used to verify this concept.

\subsection{Time}

Time is a unique characteristic for all three-dimensional geometric mass (Smolin, 2017). It is the parameter that describes the change of all materials through- 
out their existence after their creation from energy. Time is a unique entity of the three-dimensional Universe, result from the rational perceptual cognition and technological creations of humankind, and it is the tool and the method to measure the process of existence of all the natural and artificial objects.

In multi-dimensions deprived of any mass, the concept of time would not exist, for time is a unique property that is massless, and it describes the change of real matters and the three-dimensional Universe. In zero-dimensional space without real matters, time would either be zero, infinitely large or does not exist, while space would still exist, although not in a three-dimensional geometric form. All reflections over the Essence of the Universe and humankind must take into account this problem, otherwise, they would become faulty.

\section{Theories in Modern Theoretical Physics}

\subsection{Theory of Relativity}

Einstein discovered the inseparable and closely interrelated relationship between mass, time, and space, and he used the speed of light to relate mass to energy (Einstein, 2018). This discovery of Einstein is one of the greatest discovery on the Essence of the three-dimensional Universe. Furthermore, when Einstein proposed that gravitation is the manifestation of curved space-time, it confirmed that space is more than only three-dimensional, and was later sequenced following the postulation of the M-theory (Einstein, 2018; Greene, 2018). In the multi-dimensional Universe, there is no mass, nor numbers, for numbers were a creation of humans, similar to time. In the three-dimensional Universe, the change in energy and information lead to the respective change in the occupied space and time by matters. Since space and time cannot be curved, their smallest unit is zero (Greene, 2018).

The four-dimensional space-time is a notion within the three-dimensional Universe, and to study the Essence of the Universe, it is necessary to eliminate the factor of time because time is not absolute (Smolin, 2017). The theory of relativity, although a great theory in itself that explains the three-dimensional Universe and connects the three-dimensional Universe with the zero-dimensional Universe, is not the theory that explains the Truth of the Universe, because it can only adapt to particles with a mass. The scientificity of its unveiled Truth equals to the Newtonian mechanics and the electromagnetism developed by the Scottish scientist James Maxwell. These theories are all part of what defines the three-dimensional Universe, but they are not the Theory of Everything.

\subsection{Quantum Mechanics}

Quantum mechanics had led to significant development in physics and brought numerous progress in technology innovations. It also brought humans perceptive into the origin of matter by discovering the unique stage during the creation of matters. Furthermore, it discovered nuclear power and a third kind of energy close to the fundamental energy of the zero-dimensional Universe (Liu, 2019). 
With the advent of quantum mechanics, scientists were able to discover the chaotic state of the Universe at the infinitely small moment after the Big Bang (Greene, 2018). These discoveries, over the years, sparkled the reflection on the origin of matter in the circle of philosophy and religions, such as described in the prominent Metaphysics postulated by Aristotle from the Axial Age (Liu, 2017; Liu, 2019).

The origin of quantum mechanics started when Max Planck discovered the boundaries of the interchange between mass and energy and had set a limit to the mass and energy of the three-dimensional Universe (Penrose, 2013). During his research in the relationship between the light spectrum and heat energy in the metallurgy field, Planck discovered the initial building blocks of matter, the energy quantum.

Following the works of Planck, other scientists such as Niels Bohr and Paul Dirac studied in detail the energy quantum proposed by Planck and confirmed the theory that mass comes from energy and ends in energy (Hawking, 2011). Amongst the theories that described the relationship between mass and energy are the theory on the duality of wave-particle, Heisenberg's uncertainty principle, and quantum entanglement (Hawking, 2011). The unique characteristic of quantum particle where it is at the same time mass-like and energy-like perfectly showed the characteristics of the Essence of the Universe.

The world of quantum physics is filled with dialectical thinking, for every particle in the quantum world, it has a respective anti-particle, such as electrons and positrons, fermions and bosons, matters and anti-matters, and even symmetry and asymmetry. In the Universe, the opposites are not necessarily material-based. It is a concept as the opposites could well be energy-based, such as yin and yang. Instead, the most important aspect is the dialectics and unification of the concept.

The author did not found any apparent flaws in the theories of quantum mechanics on the Essence of the Universe. When relating it to the theory of relativity, however, theories in quantum mechanics showed some discrepancies with theory of relativity. The theory of relativity, similarly, is formulated for the three-dimensional Universe, and it fails to describe the multi-dimensional Universe. To this day, the greatest minds are still bounded in the geometrical three-dimensional Universe by mass, light, and time. In this three-dimensional Universe, quantum mechanics cannot unify with the theory of relativity. However, at a zero-dimensional Universe, they are consistent with each other. In the zero-dimensional space, there would exist no matters, dimension, or time. The only being in existence within this space is the energy, which includes the fundamental energy and intelligent energy. Since in a zero-dimensional space, there would be no notion of distance and time, correspondingly, the concept of the speed of light would be no more, which leads to the unification of the theory of relativity and quantum mechanics. Because particle carries both the properties of energy and matter, in a zero-dimension, it would exhibit phenomena beyond the 
concepts of current physics, such as travel at a speed faster than light.

When scrutinized, the theory of relativity cannot unify with quantum mechanics in certain aspect (Greene, 2018; Hawking, 2011). The theory of relativity is the scientific theory that links the three-dimensional Universe with mass, whereas quantum mechanics is the science that links materials to the Essence of the Universe, and physics to the multi-dimensional Universe (Penrose, 2013; Smolin, 2017; Xu, 2010). Furthermore, the theory on the Essence of the Universe in quantum mechanics involved not only scientific questions, but religious question as well, and not the theory on the Essence of any specific religion, but the theory to all religions (Hawking, 2011). In addition to its involvement in the Essence of mass, information, intelligence, and spirit, the core ideologies in oriental knowledge and the philosophical ideologies of the West, such as the First Philosophy, and Metaphysics are also alluded in theories of quantum mechanics. Hereby, the author hopes the philosophy community to unify the perception of the Universe between modern physics and the classical philosophical ideology, and once again helps the humankind in their reflections and their survival in the Universe, mutually with the assistance of modern physics.

The quantum mechanics has been a tremendous leap forward for humankind, and all of the mathematical derivations and scientific verification to date on the theories of quantum mechanics turned back positive. Quantum mechanics is also a key factor in the development and success of the third industrial revolution. For example, the theory of atomic energy creation has revolutionized humankind's perception of the application of energy and the Essence of energy. With the progress of quantum mechanics and the advent of the atomic age, it brought forth the fate-changing reflection upon humankind, that is when humankind becomes fully proficient in the application of the atomic energy, peace then becomes the only viable alternative or humankind risks its demise.

The revelation given by the study of cosmology and the theory of relativity is that human races live within a community of shared destiny. In the vast Universe, humankind has to work and function as an entity to survive.

The creation of computers and information network lifted the last veil for humankind to become a god of themselves. Materially, humankind now possesses the ability to create life. However, spiritually, humans stay humans, and if humans do not achieve breakthroughs in aspects of mind and spirit, then humans with the capability of God might become the devil. The creation of computers set a fundamental question on the Essence of cosmic information and human, that is, what is the essential difference between humans and computers.

In the perspective of philosophical reflection, quantum mechanics are not perfect. For example, in the theory of the weak force, the weak force participates in the aggregation of matters, but at the same time, it also contains the information on the cyclic growth and decline of matters. Thus, the term weak force might not be the best definition to describe this process. Higgs bosons, on the other hand, are particles that carry the cause of all other particles, and they 
should be regarded as pure information, not solely as physical particles.

The biggest philosophical, or conceptual imperfection of quantum mechanics is the expression of the absolute void that is not completely separated from the notions of mass and time. In philosophy, the Eastern philosophy described this concept of the void in detail more than two millennia ago (Feng, 2013; Graham, 1990; MacDonald, 2017; Rutt, 1996). It only lacks a "translation" in the current scientific terms. In addition to Eastern philosophical ideologies, the Classical Greek philosophical ideologies are also waiting for the development of scientific methods such that humankind can finally have tools to unscramble the wisdom of their ancestors.

In this new age of technological progress, humans need the progress of science, as well as religions, and philosophy to reflect on its future.

\subsection{M-Theory}

The M-theory is a great mathematical theory that explores the Essence of the three-dimensional Universe. It used the mathematical models to fully explore the origin of materials and the Essence of the three-dimensional matter. However, the M-theory is not the ultimate theory because its initial mathematical model included the factors of matter and time (Greene, 2018).

Initially, the Universe itself is composed of the void, a non-matter zero dimension with nothing but energy, in the form of yin and yang, and information. Under specific circumstances, the Universe can produce multi-dimensional space where space of different dimensions encompasses their respective existence and dynamics.

With the birth of matter, then came the three-dimensional space, the myriad things, and humans. Humans, with their powerful information processing capabilities, became the king of all things. Humans then utilized their information processing capability and actions to explore the Universe and the myriad things, and they have been creating all the artificial beings along the path, including matters, energy, and information. Humans are also the creator of all concepts, whether scientific, religious, or philosophic.

Over two thousand years ago, human ancestors had reached the Essence of the Universe and humankind. However, In the West, the focus of reflections and explorations had been shifted toward the materially three-dimension world following Socrates (c. 469 - c. 399 BC) (Thilly, 1914). The Eastern philosophy, however, kept its focus on the perception of the void, as their ancestor had done millennia ago ( $\mathrm{Hu}, 2012)$.

With the progress of technology, humans gained access to advanced computer simulation. From the results of a detailed simulation of the three-dimensional Universe, mass only represents $4.9 \%$ the space occupied in the three-dimensional Universe, with the remaining $95.1 \%$ occupied by the seeming vacuum or void (Sebastian, 2014). From the perspective of three-dimensional space, this conclusion is scientifically meaningful and important. However, from the pers- 
pective of the multi-dimensional Universe, such as the zero-dimensional space mentioned above, the results would be different, as only the void, without matters, exists in this dimension.

\section{Discussion on the Essence of Matters, Humanity, and the Universe}

\subsection{The Essence of Matters and Humanity}

A representation of the Essence of matters is shown in Figure 1, and that of humanity in Figure 2. All maters came from particles laden with unique information that result in the formation of myriad things. These particles are the origin of all molecular matters, including humans, and the unique information remained hidden inside the atoms or molecules. After they have been experiencing the extreme environment throughout the beginning of the three-dimensional Universe and that the Universe has reached a harmonious state of yin and yang, the information hidden in these particles started to awaken to forms atoms, the atoms then form molecules, or further into cells, depending on the final type of matters formed. Cells can further divide into plant cells, animal cells, and human cells. The information stored in the particles that ultimately form these three types of cell are different, amongst them, the cause hidden within the human cells are exotic even in the Universe, and they are the reason why humans are amongst the intelligent matters.

The Universe especially created a type of intelligent matter that can perceive, recognize, understand, and represent the Universe. To date, the only intelligent matter discovered is humans. For the Universe to create and define an intelligent matter, the author tentatively proposes that it must exhibit the following criteria:

1) The intelligent matter is a living mass formed from the combination of the information of the void and the host organism. The information controls the host organism, and once the information leaves the living host to return to the void, the life of the host ends and transforms into an ordinary matter, while the energy and information once carried by the host dissipate into the void.

2) The intelligent matter must have an unparalleled capability in the perception, process, and transmission of information. It must also have intelligence, be capable of thinking, action, creation, and destruction.

3) The intelligent matter must have an intense spontaneous urge to search for the source of survival energy, or the First Energy, such as food, to transform it. It must also exhibit a strong will to survive.

4) The intelligent matter must exhibit a strong spontaneous urge to find and mate with a partner of the opposite sex to guarantee the reproduction and continuation of the specie. It must also be capable to artificially replicate natural energy, such as electrical and thermal energy, for its survival. The author calls this energy the Second Energy.

5) The intelligent matter must have a balanced nature of yin and yang, and have the ability of dialectic reflection with its wisdom. 


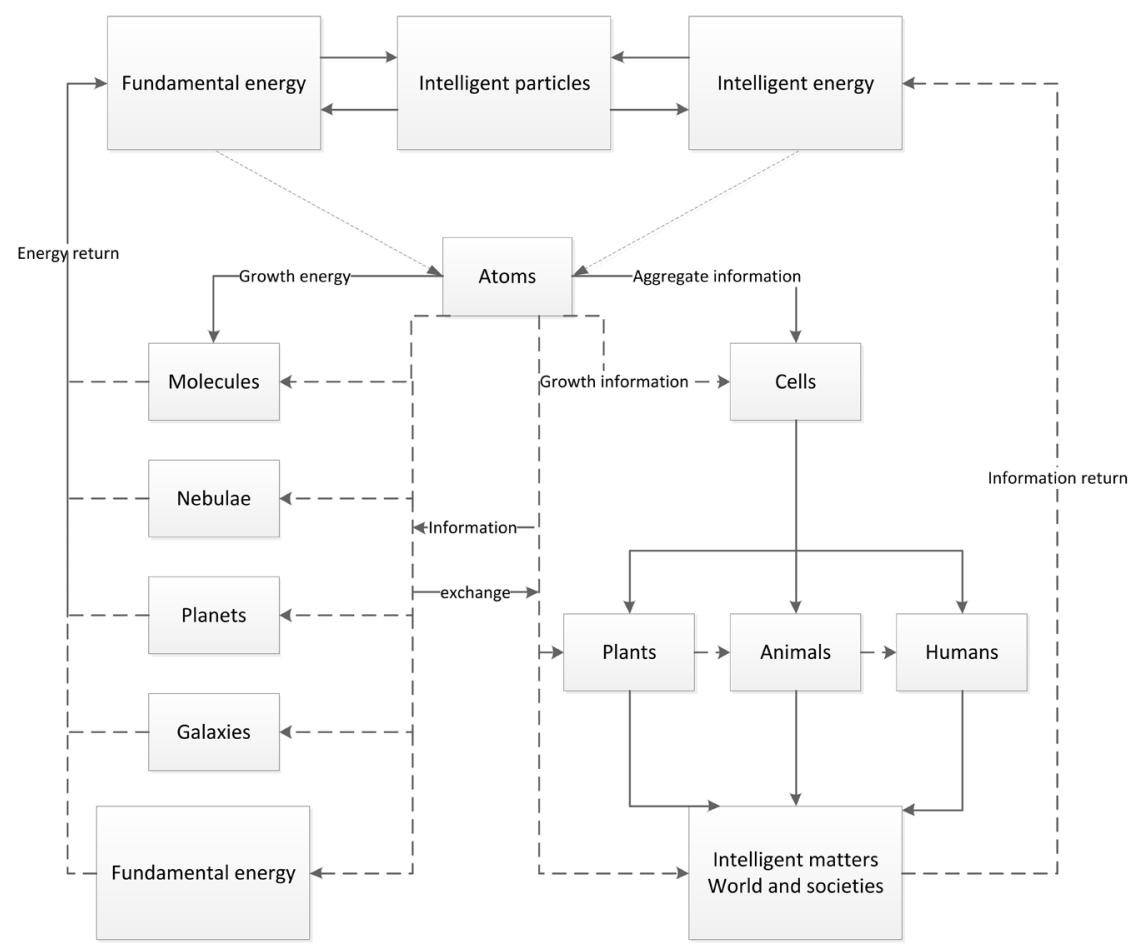

Figure 1. The Essence of matters.

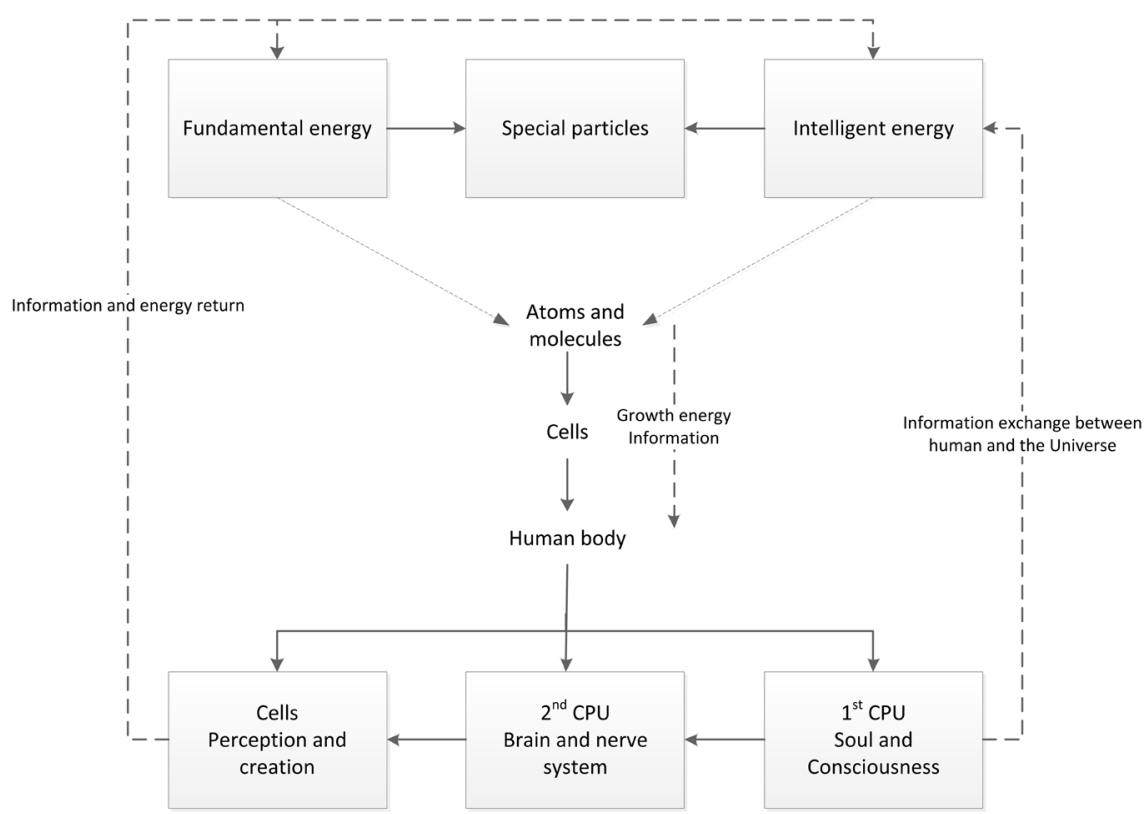

Figure 2. The Essence of humanity.

6) The intelligent matter must have the ability to communicate with the Universe, and have a strong self-centered personality, whether that is material-centered or spirit-centered.

In the design and creation of intelligent matter, the Universe did not act on a subjective way, but rather a result of from a series of natural and involuntary processes. Intelligence is a result of countless trial and error, and similar to other 
matters, intelligent matter is a mature product of the Universe. In this threedimensional Universe, intelligence probably has been creating and destroying infinite times.

Humans, created by the Universe itself, are the ultimate computer. Table 1 shows the comparison between human brain and computer.

Due to the nature of computers compared to the human brain and its limitation listed above, a computer created by humans can never replace the "computer" created by the Universe, as humans are the god of the future created by the Universe.

Humans have been starting to show some godhood both scientifically and materially, and these traits are the results from the progress of modern physics. However, humans are lacking spiritually and mentally. Within a human's soul, humanity, godhood, and animality coexist, and the condition for the dominant trait to manifest is survival. To reach godhood, human needs to recognize the Essence of humanity and the Universe, to well understand humanity itself, to perceive the cosmic dialectic thinking, to solve survival conflicts, and to live in peace and harmony. One must keep in mind that the determining factors that influence human survival are the Universe and humanity itself, and to guide it on the correct path needs the reflections and actions of the philosophy and religious community.

\subsection{Essence of the Universe}

From the scientific standpoint, the general theory of relativity proposed by Einstein describes the relationship between matters and time in a three-dimensional Universe, and it can be regarded as the scientific truth on the three-dimensional Universe. However, in the zero-dimensional space proposed by the author, or the multi-dimensional space proposed by quantum mechanics, there would be no notion of time because matters do not exist. Hence, the theory of relativity cannot be used to explore the Essence of the Universe.

The Proper Universe, or the Universe that encompasses everything known today, including the zero dimensions, originated from the void, which is a completely empty space deprived of any dimension and time. In Eastern philosophy, it is called kong and $w u(\mathrm{Hu}, 2012)$. It was this unique characteristic that subsequently gave birth to an infinite amount of multi-dimensional Universe, including the three-dimensional Universe. Figure 3 shows a detailed explanation on the Essence of the Universe. Since the Proper Universe is zero-dimensional, the three-dimensional Universe that humans live in could be a flexible pocket Universe that exists within this Proper Universe that is big without a border, small without a limit. It might also be only one of the infinite other multiverses that exist in the Universe, because if one Universe could be born, then infinite others could as well or none at all. In the three-dimensional Universe, humankind came to live, so could be infinite other kinds of intelligent species or none at all. From statistical studies, even within the known local pocket Universe, intelligent species similar to humankind must exist. 


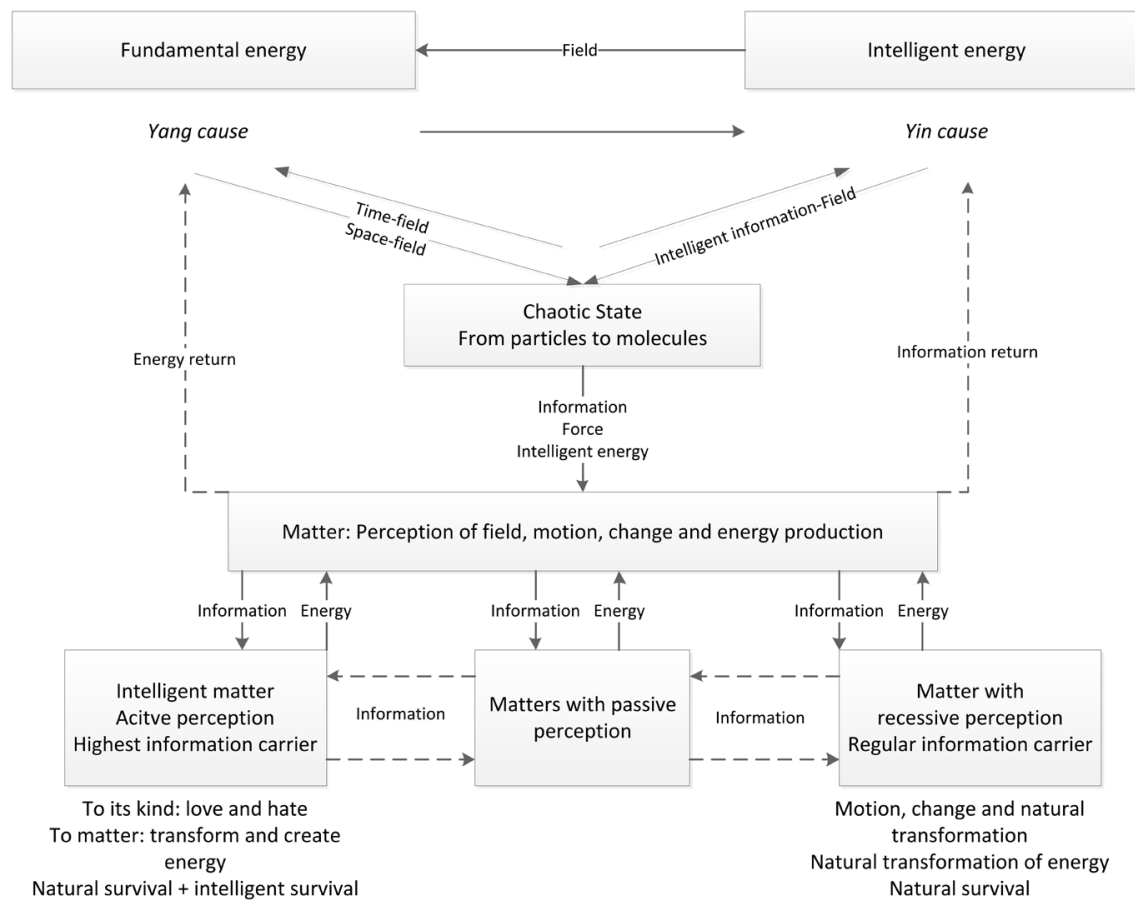

Figure 3. The Essence of the Universe.

Table 1. Comparison between human brain and computer.

\begin{tabular}{lll}
\hline Type & Human & Computer \\
\hline Creator & Universe & Human \\
Function & Unlimited, controlled by thought & Limited, controlled by human \\
$\begin{array}{l}\text { Energy } \\
\text { Source }\end{array}$ & $Q i$ & Electricity \\
Conduct & $\begin{array}{l}\text { Meridian system }(\text { Ex nihilo }) \text { and nervous } \\
\text { system }\end{array}$ & Yes \\
& $\begin{array}{l}\text { Cells with perceptive ability and each cell } \\
\text { can serve as an independent information } \\
\text { processor }\end{array}$ & $\begin{array}{l}\text { Non-active perceptive molecules } \\
\text { made from ordinary matters }\end{array}$ \\
Main Frame & Soul (void) + brain (real materials) & $\begin{array}{l}\text { Processor (real materials) }+ \text { artificial } \\
\text { intelligence }\end{array}$ \\
CPU & &
\end{tabular}

From the philosophical perspective, on the other hand, early Eastern philosophers gave many names and descriptions to the Universe. Laozi mentioned that "The nameless is the boundary of Heaven and Earth. The named is the mother of creation" (MacDonald, 2017). Buddha said the Universe is formed from Sünyatā, or kong, and described the Universe using Tri sahasra mahā sahasra lokadhătu, or the trichiliocosm, describing the Universe as a billion-fold world (Thera, 1995). How did the past saints perceive the Universe remained a mystery, but the author believed that they obtained enlightenment by reaching a complete passive immersive concentration.

The concept of kong and $w u$, in numerous ancient Chinese ideologies, is re- 
ferred to as wuji (Feng, 2013; Hu, 2012; MacDonald, 2017). Wuji is an important concept in Daoism. From Daoism texts, Wuji is infinite, without limit, and it does not contain any matters, and it is timeless. From the scientific perspective, the concept of wuji is very similar to the theory zero dimension described in earlier sections. Hence, the author named this dimension as the dimension of dao, which in Daoism texts means the beginning of everything (MacDonald, 2017). A graphical representation of wuji is shown in Figure 4.

The existence within this zero-dimensional void is a harmonious mix between the fundamental and intelligent energy. It is represented by the concept of yin and yang in taiji, as shown in Figure 5.

Within the void, there exists a dynamism that originated in the primordial Universe or the conflicts of yin and yang. This process is the conflict of wuxing, a process of generating and overcoming interactions between the five conceptual elements of metal, water, wood, fire, and earth is shown in Figure 6. The generation interactions are described by metal generates water, water generates wood, wood generates fire, fire generates earth, and earth generates metal. The process of overcoming interactions, on the other hand, it represented by metal overcomes wood, wood overcomes earth, earth overcomes water, water overcomes fire, and fire overcomes metal.

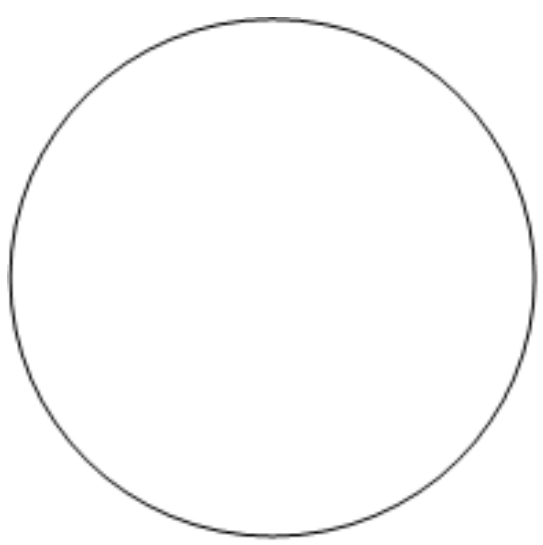

Figure 4. The Essence of the void Universe-Wuji.

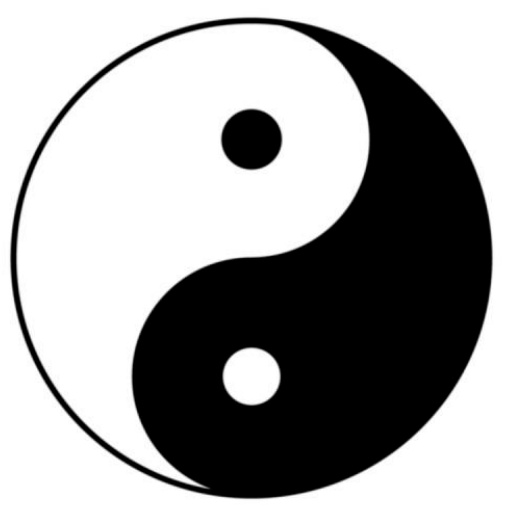

Figure 5. The Essence of the Universe-Taiji. 


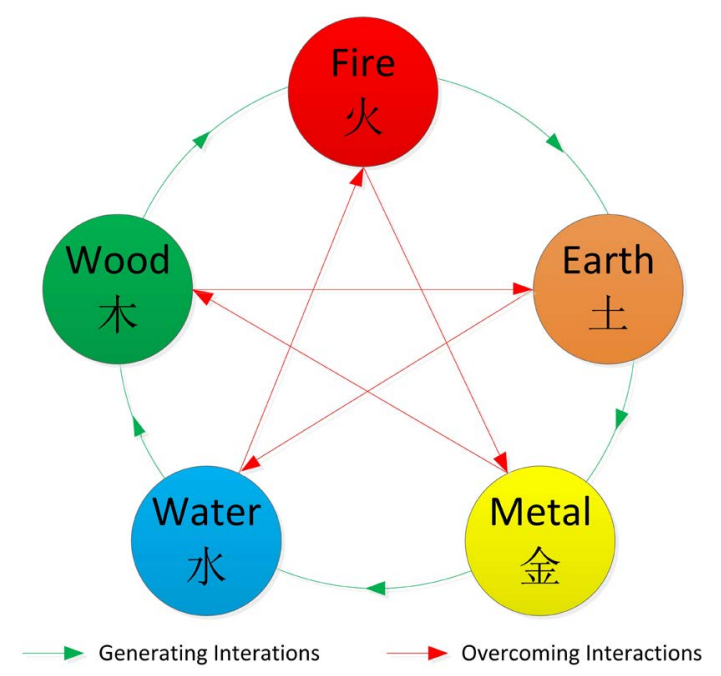

Figure 6. The Essence of the cosmic dynamismThe wuxing interactions.

In respect to the $\mathrm{M}$ theory on the definition of dimensions in the Universe, the author previously named the fourth-dimension as the $0^{+}$dimension and its energy contained as the fundamental energy (Liu, 2019). The fifth $0^{-}$dimension, or the dimension of change contains the intelligent, or information energy (Liu, 2019). The dimension of dao, or the sixth-dimension, is the dimension where the fundamental energy and intelligent energy, as well as the $0^{+}$and the $0^{-}$dimension entangled with each other in a fashion similar to that of yin and yang and forms a harmonious mix. However, these dimensions are all abstract dimensions, that is, a spatial dimension without any real mass. Because they are objective existence within the zero-dimensional Universe.

The only real existing Universe discovered to date is the three-dimensional Universe, a Universe filled with real matters and humans, and this dimension is possibly the highest dimension in the Universe.

\section{Summary}

Humans live in the three-dimensional Universe, itself a particular state in the Proper Universe. All the materials encompassed within this three-dimensional Universe came from the combination of the fundamental energy and the intelligent energy. What will atoms or molecules form is engraved during matter's birth from the information of a multi-dimensional Universe. As a result, myriad things are born, including humans, protegees of the Universe, and possibly numerous other intelligent species. However, Humankind cannot decide on the existence or fate of other intelligent species, or the way the Universe operates. But, it has the final words on how humans survive in the Universe. With the current progress of technology, humans are able to cope with most cosmic threats. Nonetheless, humankind still needs the work of the entire human race to guarantee its survival within the Universe. The best solution to counter the cosmic threats lies in the harmony and peace between human races, and the love and reflection 
within the human's soul. This work intends to initiate a reflection over the Essence of the Universe and humankind, whether this reflection ends in resonance or disputes.

It is now time.

\section{Conflicts of Interest}

The author declares no conflicts of interest regarding the publication of this paper.

\section{References}

Einstein, A. (2018). Melt Weltbild. Beijing: CITIC Press.

Feng, Y. (2013). A Brief History of Chinese Philosophy. Beijing: Beijing University Press.

Graham, A. (1990). The Book of Lieh-tzu. New York: Columbia University Press. https://doi.org/10.7312/grah93146

Greene, B. (2018). The Elegant Universe: Superstrings, Hidden Dimensions, and the Quest for the Ultimate Theory. Changsha: Hunan Science \& Technology Press Co. Ltd.

Hawking, S. (2011). The Grand Design. Changsha: Hunan Science \& Technology Press Co., Ltd.

Hu, S. (2012). Syllabus to the Chinese Philosophy History. Beijing: Zhonghua Book Company.

Leibniz, G. W. (2001). Fourth Paper. In S. Clarke (Ed.), A Collection of Papers, Which Passed between the Late Learned Mr. Leibnitz, and Dr. Clarke, in the Years 1715 and 1716 (pp. 93-107). Boston, MA: Adamant Media Corporation.

Liu, S. (2017). Revelation and Reflection on Mankind by Modern Physics Part I. Open Journal of Philosophy, 7, 435-447. https://doi.org/10.4236/ojpp.2017.74023

Liu, S. (2019). Revelation and Reflection on Mankind by Modern Physics Part II Consideration on Multidimensional Universe. Open Journal of Philosophy, 9, 72-81. https://doi.org/10.4236/ojpp.2019.92007

Liu, S. (2020). Philosophical Reflection over the Origin of the Universe. Philosophy Study, 3, 213-222.

MacDonald, J. H. (2017). Tao Te Ching: An Insightful and Modern Translation by J. H. McDonald. Qigong Vacations.org.

Penrose, R. (2013). The Road to Reality: A Complete Guide to the Laws of the Universe. Changsha: Hunan Science \& Technology Press Co., Ltd.

Porter, B. (2018). Road to Heaven-Encounters with Chinese Hermit. Chengdu: Sichuan Literature \& Art Publishing House.

Rutt, R. (1996). The Book of Changes (Zhouyi) a Bronze Age Document. Richmond, Surrey: Curzon.

Sebastian, A. (2014). Structure of Relatively Accelerating Universe. International Journal of Astronomy and Astrophysics, 4, 165-177. https://doi.org/10.4236/ijaa.2014.41015

Smolin, L. (2017). Three Roads to Quantum Gravity. New York: Hachette Book Group.

Thera, S. (1995). The Long Discourses of the Buddha: A Translation of the Digha Nikaya. Somerville, MA: Wisdom Publications.

Thilly, F. (1914). A History of Philosophy. New York: H. Holt and Company.

Xu, L. (2010). Anthology of Albert Einstein. Beijing: Commercial Press China. 